\title{
ĐỊNH VỊ ĐIỂM CHÍNH XÁC CAO CÓ GIẢI THAM SỐ ĐA TRI VÀ XỬ LÝ KẾT HỢP ĐA HỆ THỐNG VỆ TINH ĐỊNH V!̣
}

\author{
NGUYẼ̃N NGỌC LÂU ${ }^{(1,2)}$, NGUYẼ̃N TH! THANH HƯONG ${ }^{(3)}$ \\ ${ }^{(1)}$ Bộ môn Địa Tin học, Trường Đại học Bách khoa TP. HCM \\ ${ }^{(2)}$ Đại hoc Quốc gia TP. HCM \\ ${ }^{(3)}$ Viện Khoa học Đo đạc và Bản đồ
}

\section{Tóm tắt:}

Gần đây, định vị điểm chính xác (Precise Point Positioning - PPP) đã được nhũng cải thiện đáng kể nhờ vào các sản phẩm PPP của một số dịch vu khác nhau. Nhũng dịch vu này cung cấp các sản phẩm PPP khác nhau ở khả năng giải đa trị (Ambiguity Resolution - AR) và các hệ thống vệ tinh định vị. Chúng tôi muốn nghiên cứu xem các hệ thống ngoài GPS bổ sung và việc giải đa trị sẽ ảnh huởng nhu thế nào đến độ chính xác của PPP. Bằng cách xủ lý dũ liệu GNSS 24 h tại 47 trạm thuoòng trưc IGS với các chọn lựa khác nhau về AR và sụ kết hợp của các hệ thống vệ tinh, kết quả của chúng tôi cho thấy không có sự cải thiện về độ chính xác định vị đối với giải pháp tĩnh $24 h$ (2.1, 2.1, 5.8mm ở các thành phần huớng Bắc, Đông và độ cao). Tuy nhiên, sự kết hơp của GPS + GLONASS + GALILEO với GPS AR mang lại độ chính xác tốt nhất ở định vị tùng thời điểm (6.6, 6.2, 20.2mm). So với truờng hợp chỉ dùng GPS với AR, tùy chọn này có thể cái thiện độ chính xác lên đến $26 \%$ trên thành phần độ cao.

\section{Giới thiệu}

Định vị điểm chính xác cao (Precise Point Positioning - PPP) là phương pháp định vị xử lý đồng thời các trị đo pha sóng tải và trị đo giả cự ly từ một máy thu GNSS duy nhất, cùng với việc áp dụng các sản phẩm chính xác về quỹ đạo và đồng hồ vệ tinh. Tổ chức uy tín nhất cung cấp các sản phẩm đồng hồ và quỹ đạo GNSS chính xác cho người dùng dân sự là Dịch vụ GNSS Quốc tế (International GNSS Service -IGS) [5].

PPP truyền thống bắt đầu vào cuối những năm 1990 [1], khi coi tham số đa trị trong trị đo pha là một ẩn số bổ sung để ước tính cùng với các ẩn số khác như tọa độ máy thu, độ trễ tầng đối lưu, v.v. Bằng cách này, giá trị ước tính của tham số đa trị là giá trị thực. Tọa độ máy thu nhận được trong trường hợp này được gọi là nghiệm trôi.

Việc giải đa trị cho PPP (PPP-AR), tức là tìm giá trị nguyên chính xác cho các tham số đa trị, chưa thể thực hiện được vì chúng ta không thể tách độ trễ tín hiệu của phần cứng vệ tinh khỏi tham số này. Những nỗ lực để giải đa trị cho PPP bắt đầu từ năm 2005 [3, 4], khi một số nhà nghiên cứu và tổ chức khoa học tìm cách xác định độ trễ của phần cứng vệ tinh thông qua mạng lưới các trạm GNSS cốt lõi và truyền số hiệu chỉnh này tới người dùng bằng một trong vài cách khác nhau [12]. Nếu các tham số đa trị có thể được tách biệt và thay thế bằng các số nguyên chính xác, điều này có thể tăng độ hội tụ PPP để cung cấp độ chính xác $\mathrm{cm}$ ở mặt bằng chỉ trong vài chục phút hoặc thậm chí chỉ vài phút kể từ khi bắt đầu đo. Nghiên cứu trước đây của chúng tôi về $\mathrm{PPP}-\mathrm{AR}$ [15] đã chỉ ra rằng PPP-AR có thể cải thiện độ chính xác định vị lên đến 30-38\% ở thành phần hướng Bắc khi so sánh với $\mathrm{PPP}$ không có AR.

Trong những năm gần đây, số lượng các hệ

Ngày nhận bài: 5/9/2021, ngày chuyển phản biện: 9/9/2021, ngày chấp nhận phản biện: 15/9/2021, ngày chấp nhận đăng: 18/9/2021 
thống vệ tinh định vị đã tăng lên nhanh chóng. Ngoài GPS, những hệ thống khác như GLONASS, GALILEO, BEIDOU đều có sẵn cho người dùng trên toàn thế giới. PPP không có AR với đa hệ thống vệ tinh có khả năng cung cấp độ chính xác tốt hơn $1 \mathrm{~cm}$ khi đo tĩnh 24 giờ và tốt hơn 1 decimet khi đo động [16, 17, 18].

Trong bài báo này, chúng tôi muốn nghiên cứu khi thêm vào các hệ thống vệ tinh ngoài GPS và việc giải đa trị sẽ ảnh hưởng đến độ chính xác của PPP như thế nào? Khi kết hợp đồng thời việc giai đa trị và xử lý đa hệ thống vệ tinh GNSS, độ chính xác của PPP có thể được cải thiện như thế nào? Chúng tôi trình bày tóm tắt về phương pháp giải đa trị cho PPP bằng cách sử dụng các sản phẩm PPP-AR trong phần 2 . Phần 3 giới thiệu cách lựa chọn các trạm đo GNSS để đánh giá độ chính xác của PPP-AR. Kết quả và kết luận sẽ được trình bày trong phần 4 và 5 của bài báo.

\section{Các sản phẩm PPP-AR cho việc giải đa trị và xử lý đa hệ thống vệ tinh GNSS}

Chúng ta có thể giải đa trị cho các trị đo pha sóng tải từ một máy thu duy nhất bằng cách áp dụng các sản phẩm vệ tinh bổ sung, trong đó thành phần lẻ - đại diện cho độ trễ phần cứng vệ tinh - đã tách biệt với các tham số đa trị trong việc xử lý một mạng lưới GNSS. Một trong những phương pháp tạo ra các sản phẩm như vậy bằng cách lấy trung bình các phần lẻ của các tham số đa trị ước lượng được ở dạng số thực. Một phương pháp khác là ước tính độ lệch đồng hồ máy thu trong các trị đo giả cự ly và trị đo pha một cách độc lập bằng cách cố định trước tham số đa trị vào các số nguyên.

Theo [12], hiện có ba cách tiếp cận để tạo ra các sản phẩm PPP-AR: phần lẻ chu kỳ (Fractional Cycle Bias - FCB), đồng hồ khôi phục nguyên (Integer Recovery Clock - IRC) [10] và đồng hồ tách rời (Decoupled Clock - DC) [8]. Đối với người dùng PPP, mô hình toán học của ba phương pháp trên là tương tự nhau. Các sản phẩm PPP-AR này khác nhau nhưng chứa cùng một thông tin. Do đó chúng có thể được chuyển đổi từ dạng này sang dạng khác. Tổng quan về các nhà cung cấp khác nhau và sản phẩm của họ được thể hiện trong hình 1 .

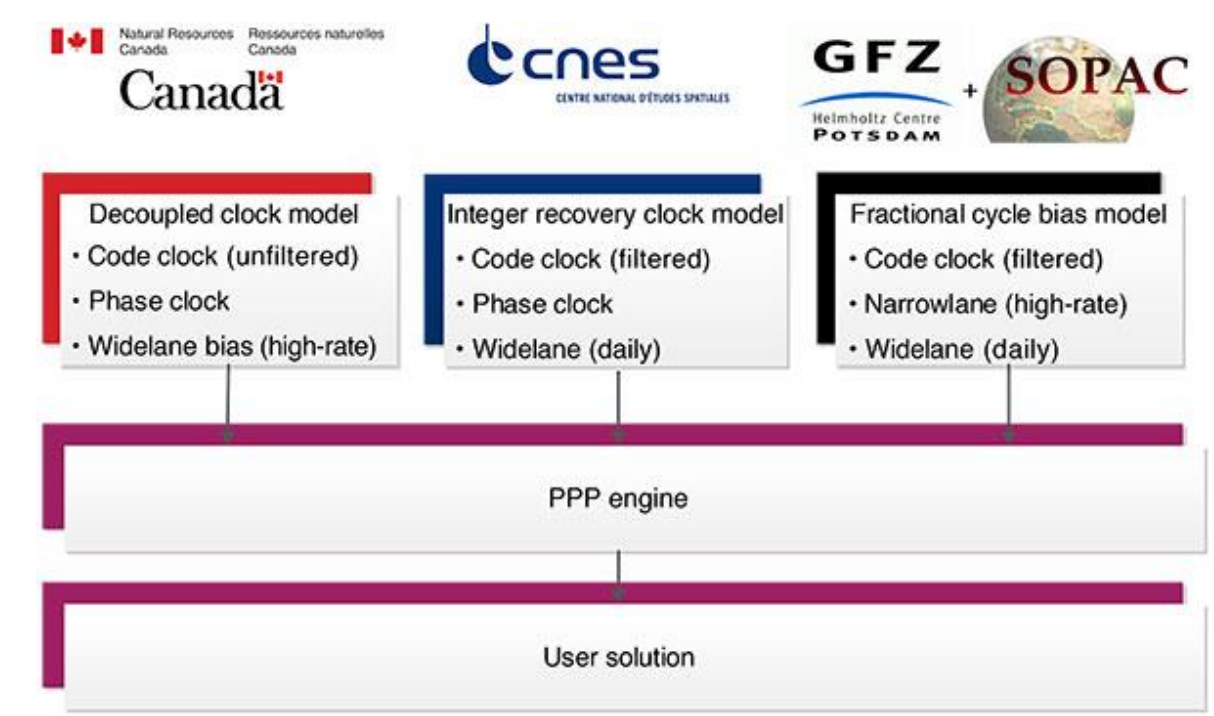

Hình 1: Các nhà cung cấp sản phẩm PPP-AR khác nhau (chấp nhận tù [12])

Cần nhấn mạnh rằng trước năm 2019, tất cả các sản phẩm PPP-AR chỉ phục vụ cho GPS. Do đó, việc giải đa trị trong PPP trước 2019 chỉ có thể thực hiện được đối với các trị đo pha GPS.
Cheng và Wang [11] đã so sánh kết quả PPP khi sử dụng các sản phẩm PPP-AR dưới dạng IRC và $\mathrm{FCB}$ và kết luận rằng mặc dù các phương pháp này tương tự nhau về lý thuyết nhưng trên 
thực tế PPP-AR dựa trên IRC hoạt động tốt hơn một chút so với PPP-AR dựa trên FCB do sai số hệ thống của ước tính FCB.

Trong bài báo này, chúng tôi sử dụng sản phẩm IRC của Trung Tâm Không gian Quốc gia Pháp (Center National d'Etudes Spatiales CNES). CNES hiện là một trong những trung tâm đóng góp vào sản phẩm của GPS, GLONASS và GALILEO và hiệu chỉnh đồng hồ cho IGS. Việc áp dụng các sản phẩm CNES vào PPP-AR được mô tả chi tiết trong bài báo [10]. Sau đây chúng tôi chỉ tóm tắt những điểm chính

Trị đo pha sóng tải không bị ảnh hưởng tầng điện ly giữa máy thu i và vệ tinh $\mathrm{k}$ được biểu thị bằng

$$
\Phi_{i, 3}^{k}=\rho_{i}^{k}+T_{i}^{k}+\delta m_{i, 3}^{k}+c\left[d t_{i}-d t^{k}\right]+c\left[\delta_{i}-\delta^{k}\right]+\lambda_{1} b_{i, 3}^{k}+\varepsilon_{i, 3}^{k}
$$

Trong đó:

$+\rho_{i}^{k}$ là khoảng cách hình học giữa máy thu

i và vệ tinh $\mathrm{k}$

$+T_{i}^{k}$ là độ trễ đối lưu

$+\delta m_{i, 3}^{k}$ là ảnh hưởng của đa đường

$+\mathrm{dt}_{\mathrm{i}}$ và $\mathrm{dt}^{\mathrm{k}}$ là sai số đồng hồ máy thu i và vệ tinh $\mathrm{k}$

$+\delta_{\mathrm{i}}$ và $\delta^{\mathrm{k}}$ và độ trễ phần cứng của máy thu $\mathrm{i}$ và vệ tinh $\mathrm{k}$

$+\varepsilon$ là độ nhiễu máy thu

$+b_{i, 3}^{k}$ là tham số đa trị. Đối với mục đích giải

đa trị, $b_{i, 3}^{k}$ được phân tích thành dạng

$$
b_{i, 3}^{k}=\frac{f_{L_{1}}}{f_{L_{1}}+f_{L_{2}}} N_{i, 1}^{k}+\frac{f_{L_{1}} f_{L_{2}}}{f_{L_{1}}^{2}-f_{L_{2}}^{2}}\left(N_{i, 1}^{k}-N_{i, 2}^{k}\right)
$$

$\left(N_{i, 1}^{k}-N_{i, 2}^{k}\right)=N_{i, w}^{k}$ được gọi là tham số đa trị dải rộng, còn $\left(N_{i, 1}^{k}\right)$ - tham số đa trị dải hẹp. Tham số đa trị dải rộng thường được ước lượng bằng cách dùng tổ hợp Melbourne-Wubbena

$$
\Phi_{i, 1}^{k}-\Phi_{i, 2}^{k}-\frac{f_{L_{1}} P_{i, 1}^{k}+f_{L_{2}} P_{i, 2}^{k}}{f_{L_{1}}+f_{L_{2}}}=N_{i, w}^{k}+\delta N_{i, w}^{k}
$$

$+\Phi_{1}$ và $\Phi_{2}$ là trị đo pha trên tần số $\mathrm{f} 1$ và f2

$+\mathrm{P}_{1}$ và $\mathrm{P}_{2}$ là trị đo giả cự ly trên tần số $\mathrm{f} 1$ và f2

$+\delta N_{i, w}^{k}$ là số hiệu chỉnh dải rộng

Ở dạng hiệu giữa các vệ tinh, Ф3 có dạng sau:

$$
\Delta \Phi_{i, 3}^{k l} \approx \Delta \rho_{i}^{k l}+\Delta T_{i}^{k l}-c\left[d t^{k}+\delta^{k}\right]+
$$

$c\left[d t^{l}+\delta^{l}\right]+\lambda_{1} \Delta b_{i, 3}^{k l}+\Delta \varepsilon_{i, 3}^{k l}$

$\left(d t^{k}+\delta^{k}\right)$ được gọi là số hiệu chỉnh đồng hồ pha của vệ tinh $\mathrm{k}$.

Đối với các trị đo GLONASS, GALILEO và BEIDOU, chúng ta vẫn dùng cùng phương trình (4). Nhưng khác với GPS là chúng không có số hiệu chỉnh dải rộng và đồng hồ pha. Do đó không thể giải trị cho những trị đo này. Khi đó các tham số đa trị trong (4) được coi là các giá trị thực (không phải là số nguyên). Để loại bỏ sai lệch tồn tại giữa các hệ thống vệ tinh khác nhau (InterSystem Biases - ISB) khi xử lý kết hợp, chúng ta cần phải thành lập hiệu giữa các vệ tinh trong cùng một hệ thống.

IRC của CNES hiện cung cấp số hiệu chỉnh dải rộng hàng ngày và số hiệu chỉnh đồng hồ pha của các vệ tinh GPS mỗi 30 giây. Vì vậy, để giải đa trị cho GPS đồng thời với quá trình xử lý nhiều GNSS, chúng tôi thực hiện quy trình gồm 2 bước:

Bước 1: Sử dụng tổ hợp MelbourneWubbena với số hiệu chỉnh dải rộng để ước tính và giải tham số đa trị dải rộng $\mathrm{Nw}$ cho GPS. Sử dụng $\Phi 3$ với số hiệu chỉnh đồng hồ pha để ước tính tham số đa trị b3 cho GPS. Giải tham số dải hẹp N1 cho GPS dựa trên Nw và ước lượng b3.

Bước 2: Loại bỏ b3 khỏi $\Phi 3$ của GPS, đồng thời coi b3 của các trị đo khác GPS là ẩn số bổ sung để ước tính cùng với tọa độ máy thu và các tham số trễ tầng đối lưu.

Trong đó:

\section{Thu thập số liệu GNSS và xử lý PPP}




\section{Nghiên cứu}

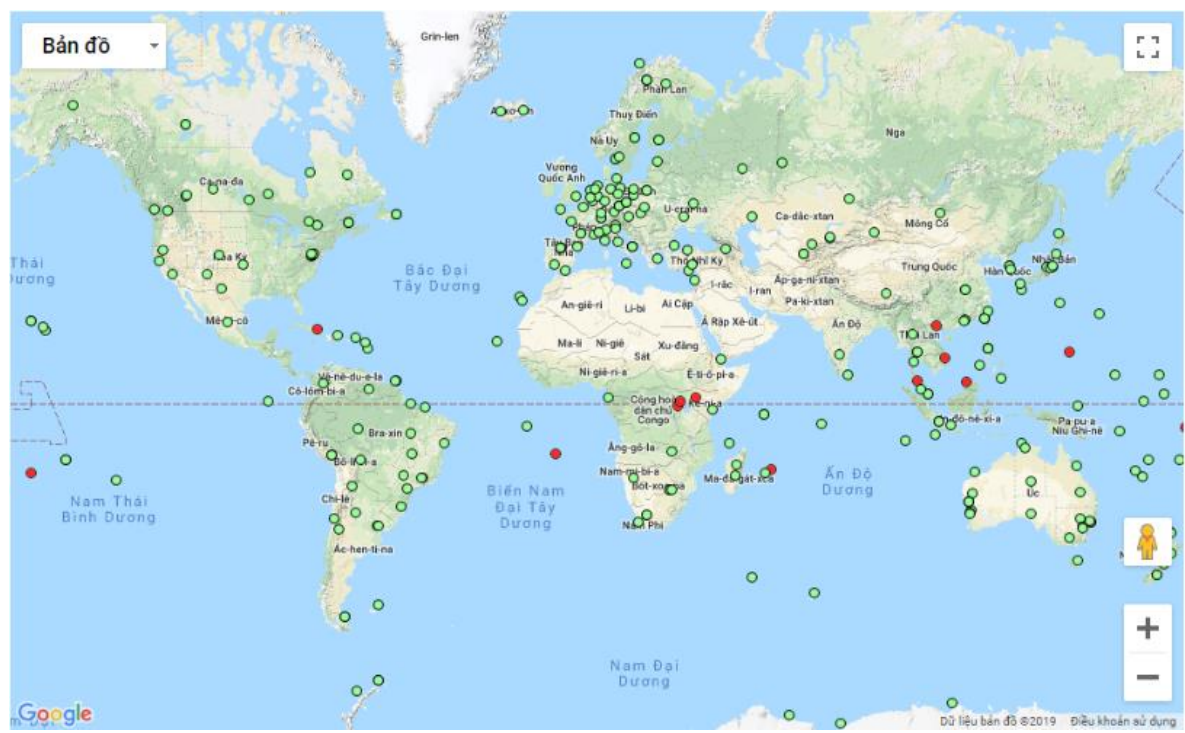

Hình 2: Vị trí các trạm đo đa hệ thống vệ tinh của IGS

Để đánh giá độ chính xác PPP một cách đáng tin cậy, tọa độ chính xác của các trạm GNSS là quan trọng và phải nằm trong cùng một hệ tọa độ với quỹ đạo vệ tinh chính xác và các số hiệu chỉnh đồng hồ. Gần đây, IGS đã thông qua một hệ tọa độ mới, được gọi là IGS14, vào ngày 2901-2017 (Tuần lễ GPS 1934) [14]. Đồng thời, kèm theo đó là một tập ăng-ten vệ tinh và ăngten mặt đất đã được cập nhật có tên là igs 14.atx. Việc chuyển đổi từ IGb08/ igs08.atx sang IGS14/igs14.atx đã được công bố vào ngày 2112-2016 bởi đại diện của các nhóm làm việc về khung tham chiếu và ăng ten IGS, cũng như các Điều phối viên của Trung tâm.

Dữ liệu của 47 trạm GNSS đo vào ngày 2108-2018 được tải xuống cùng với quỹ đạo và số hiệu chỉnh đồng hồ vệ tinh chính xác của CNES. Máy thu trang bị tại các trạm này đều là loại đa hệ thống vệ tinh (xem Hình 2). Tọa độ của các trạm được cung cấp trong IGS14 với độ chính xác vài $\mathrm{mm}$ vào thời điểm năm 2010.0. Những dữ liệu này được xử lý bằng phần mềm PPPC, do chúng tôi phát triển từ năm 2010 [7]. Phần mềm PPPC có khả năng xử lý dữ liệu GNSS ở cả hai chế độ tĩnh và động và cho nhiều hệ thống vệ tinh khác nhau như GPS, GLONASS, GALILEO, BEIDOU và QZSS. Gần đây nhất, chúng tôi đã nâng cấp PPPC với khả năng giải đa trị khi dùng các sản phẩm IRC của CNES. Một số cài đặt chung khi xử lý bằng $\mathrm{PPPC}$ được cho trong Bảng 1 .

Bảng 1: Các tham số cài đặt trong xủ lý PPP bằng PPPC

\begin{tabular}{|c|c|}
\hline Nội dung & Giá trị \\
\hline $\begin{array}{l}\text { Bản lịch và số hiệu chỉnh } \\
\text { đồng hồ vệ tinh chính xác }\end{array}$ & CNES \\
\hline Trị đo & $\mathrm{P}_{3}$ và $\Phi_{3}$ ở dạng hiệu giữa các vệ tinh \\
\hline Góc ngưỡng vệ tinh & $5^{\circ}$ \\
\hline Sai số trị đo & $\operatorname{Exp}\left(-\varepsilon / 9^{\circ}\right), \varepsilon$ là góc cao vệ tinh \\
\hline Xử lý độ trễ đối lưu & $\begin{array}{l}1 \text { tham số TZD cho mỗi } 30 \text { phút và } 2 \\
\text { gradient cho mỗi } 12 \text { giờ }\end{array}$ \\
\hline Hàm ánh xạ đối lưu & VMF1 \\
\hline Giải đa trị & $\begin{array}{l}\text { Giải đa trị dải rộng trước, dải hẹp sau cho } \\
\text { GPS. Không giải đa trị cho các vệ tinh khác } \\
\text { GPS }\end{array}$ \\
\hline
\end{tabular}


Trong hầu hết các trạm GNSS đã chọn, có rất ít trị đo $\mathrm{BEIDOU}$ và chủ yếu ở những trạm nằm trong khu vực Châu Á - Thái Bình Dương. Mặt khác, các sản phẩm của CNES vẫn chưa cung cấp quỹ đạo và đồng hồ vệ tinh chính xác cho BEIDOU. Do đó, chúng tôi quyết định bỏ qua các trị đo BEIDOU trong nghiên cứu này. Để khảo sát độ chính xác của PPP do ảnh hưởng bởi $\mathrm{AR}$ và multi-GNSS, dữ liệu cho mỗi trạm đo được xử lý theo bốn trường hợp sau:

- TH 1: chỉ dùng trị đo GPS có giải đa trị

- TH 2: dùng trị đo GPS có giải đa trị và GLONASS không giải đa trị

\section{Kết quả và phân tích}

Bảng 2: So sánh kết quả xủ lý tĩnh $24 h$

\begin{tabular}{|l|r|r|r|r|}
\hline \multirow{2}{*}{ Trường hợp } & \multicolumn{4}{|c|}{ SSTP (mm) } \\
\cline { 2 - 5 } & \multicolumn{1}{|c|}{ Bắc } & \multicolumn{1}{c|}{ Đông } & \multicolumn{1}{c|}{ Độ cao } & \multicolumn{1}{c|}{ 3D } \\
\hline GPS only with AR & 2.1 & 2.1 & 5.8 & 6.5 \\
\hline GPS with AR + GLONASS & 2.1 & 2.0 & 5.8 & 6.5 \\
\hline GPS with AR + GALILEO & 2.1 & 1.8 & 5.9 & 6.5 \\
\hline $\begin{array}{l}\text { GPS with AR + GLONASS } \\
\text { + GALILEO }\end{array}$ & 2.1 & 1.8 & 5.9 & 6.5 \\
\hline
\end{tabular}

Kết quả của bảng 2 cho thấy không có sự khác biệt về độ chính xác định vị giữa các trường hợp. Việc bổ sung thêm nhiều vệ tinh không cải thiện độ chính xác của nghiệm 24 giờ. Chúng tôi cho rằng thời gian 24 giờ là đủ dài để kết quả của tất cả các trường hợp đều hội tụ ở cùng một giá trị. Hơn nữa, việc không giải đa trị cho các trị đo và dẫn đến không thể đóng góp đáng kể vào độ chính xác cuối cùng như GPS. Kết quả của trường hợp 1 cũng giống với nghiên cứu trước đây của chúng tôi đã thực hiện vào năm 2017 [15] và Bertige và các cộng sự [6] $(2.9,2.1,6.0)$ mm hoặc Geng và các cộng sự [9] $(3.6,2.3,6.4)$ $\mathrm{mm}$.

khác GPS sẽ làm giảm độ chính xác của chúng,

Bảng 3: So sánh kết quả xủ lý tùng thời điểm

\begin{tabular}{|l|r|r|r|l|}
\hline \multirow{2}{*}{\multicolumn{1}{|c|}{ Trường hợp }} & \multicolumn{4}{|c|}{ SSTP (mm) } \\
\cline { 2 - 5 } & \multicolumn{1}{c|}{ Bắc } & \multicolumn{1}{c|}{ Đông } & \multicolumn{1}{c|}{ Độ cao } & \multicolumn{1}{c|}{ 3D } \\
\hline GPS only with AR & 8.8 & 7.5 & 27.2 & 29.6 \\
\hline $\begin{array}{l}\text { GPS with AR + } \\
\text { GLONASS }\end{array}$ & 7.0 & 6.7 & 23.2 & 25.1 \\
\hline GPS with AR + GALILEO & 7.3 & 6.8 & 23.0 & 25.1 \\
\hline $\begin{array}{l}\text { GPS with AR + } \\
\text { GLONASS + GALILEO }\end{array}$ & 6.6 & 6.2 & 20.2 & 22.1 \\
\hline Cải thiện & $25 \%$ & $17 \%$ & $26 \%$ & $25 \%$ \\
\hline
\end{tabular}


Đối với nghiệm khi xử lý từng thời điểm, kết quả của tất cả các trường hợp là khá khác nhau. Số lượng vệ tinh khác GPS tại một thời điểm đo đã làm cho nghiệm hội tụ nhanh hơn. Có thể thấy rõ rằng mức độ cải thiện độ chính xác phụ thuộc vào số lượng hệ thống vệ tinh được bổ sung. Mặc dù đóng góp ít vệ tinh hơn GLONASS, sự kết hợp của GPS + GALILEO vẫn cho độ chính xác tương đương với sự kết hợp của GPS + GLONASS, và tốt hơn GPS only. Độ chính xác cao nhất có thể đạt được với trường hợp 4 . So với trường hợp chỉ dùng GPS, thành phần được cải thiện nhiều nhất là độ cao $(26 \%)$, tiếp theo là hướng Bắc (25\%) và hướng Đông (17\%).

\section{Kết luận}

Chúng tôi đã kiểm tra độ chính xác của PPP với việc giải đa trị và đa hệ thống vệ tinh trên một tập hợp gồm 47 trạm GNSS vào ngày 21-082018 ở hai chế độ: tĩnh 24 giờ và từng thời điểm đo. Kết quả có thể được tóm tắt như sau:

- Độ chính xác của giải pháp 24 giờ là như nhau đối với tất cả các trường hợp xử lý $(2,1,1,9$ và $5,8 \mathrm{~mm}$ ở các thành phần Đông, Bắc và độ cao). Việc thêm nhiều vệ tinh khác GPS vào giải pháp không cải thiện độ chính xác của PPP. Điều này có thể thay đổi trong tương lai khi các sản phẩm PPP-AR có thể mở rộng cho các vệ tinh này.

- Độ chính xác của việc xử lý từng thời điểm phụ thuộc rõ ràng vào số lượng GNSS được sử dụng trong quá trình xử lý. Sự kết hợp giữa GPS + GALILEO cho độ chính xác tương đương với sự kết hợp của GPS + GLONASS và tốt hơn so với GPS only. Độ chính xác cao nhất có thể đạt được khi kết hợp GPS + GLONASS + GALILEO. So với trường hợp chỉ có GPS, thành phần được cải thiện nhiều nhất là độ cao $(26 \%)$, tiếp theo là hướng Bắc $(25 \%)$ và hướng Đông $(17 \%)$.

Với độ chính xác đạt được của PPP khi có $\mathrm{AR}$ và multi-GNSS, cho phép chúng ta mở rộng các ứng dụng của PPP để giám sát chuyển dịch của mặt đất ở trạng thái chậm hoặc đột ngột một cách chính xác và hiệu quả hơn so với phương pháp định vị tương đối và $\mathrm{PPP}$ truyền thống. $\mathrm{O}$

\section{Tài liệu tham khảo}

[1]. J.F. Zumberge, M.B.Heflin, D.C. Jefferson, M.M. Wattkins and F.H. Webb, 1997. Precise point positioning for the efficient and robust analysis of GPS data from large networks, Journal of Geophysical Research, Vol 102, No B3, p. 5005-5017.

[2]. Matt King, Stuart Edwards and Peter Clarke, 2002. Precise Point Positioning: Breaking the Monopoly of Relative GPS Processing, Engineering Surveying Showcase 10/2002, p. 33-34.

[3]. Ge M, Gendt G, Dick G and Zhang FP, 2005. Improving carrier-phase ambiguity resolution in global GPS network solutions, Journal of Geodesy, Vol 79, p. 103-110.

[4]. Ge M, Gendt G, Rothacher M, 2006. Integer ambiguity resolution for precise point positioning: applied to fast integrated estimation of very huge GNSS networks, Paper presented at VI Hotine-Marussi Symposium of theoretical and computational Geodesy, Wuhan 29 May-2 June 2006.

[5]. Kouba J., 2009. A Guide to using International GNSS Service (IGS) products, Natural Resources Canada, http://igscb.jpl.nasa.gov/components/usage.html

[6]. Willy Bertige, Shailen D. Desai, Bruce Haines, Nate Harvey, Angelyn W. Moore, Susan Owen, Jan P. Weiss, 2010. Single receiver phase ambiguity resolution with GPS data, Journal of Geodesy, Vol 84, p. 327-337.

[7]. Nguyễn Ngọc Lâu, Trần Trọng Đức, Dương Tuấn Việt, Đặng Văn Công Bằng, 2010. Automatic GPS precise point processing via internet, Report of ministry level project B201030-33.

[8]. Junbo Shi, 2012. Precise Point Positioning Integer Ambiguity Resolution with Decoupled Clocks, PhD Thesis at the UNIVERSITY OF CALGARY, Canada. 
[9]. Jianghui Geng, Chuang Shi, Maorong Ge, Alan H. Dodson, Yidong Lou, Qile Zhao, Jingnan Liu, 2012. Improving the estimation of fractional-cycle biases for ambiguity resolution in precise point positioning, Journal of Geodesy, Vol 86, p. 579-589.

[10]. D. Laurichesse, 2012. Phase Biases Estimation for Undifferenced Ambiguity Resolution, PPP-RTK \& Open Standards Symposium, March 12-13, 2012, Frankfurt.

[11]. Shuyang Cheng and Jinling Wang, 2016. Ambiguity Resolution (PPP-AR) For Precise Point Positioning Based on Combined GPS Observations, International Global Navigation Satellite Systems Association IGNSS Conference 2016, Colombo Theatres, Kensington Campus, UNSW Australia $6-8$ December 2016.

[12]. Garrett Seepersad and Sunil Bisnath, 2016. Examining the interoperability of precise point positioning products, GPS World, Vol 27, No 3, 50-56, March 8, 2016.

[13]. Garrett Seepersad, Simon Banville, Paul Collins, Sunil Bisnath, François, Lahaye, 2016. Integer satellite clock combination for Precise Point Positioning with ambiguity resolution, Proceedings of the 29th International Technical Meeting of The Satellite Division of the Institute of Navigation (ION GNSS+ 2016), p. 2058 - 2068.

\section{Summary}

\section{Precise point positioning accuracy with ambiguity resolution and multi-gnss}

Nguyen Ngoc Lau, Department of Geomatics Engineering, Ho Chi Minh City University of Technology, Vietnam. Vietnam National University Ho Chi Minh City, Vietnam

Nguyen Thi Thanh Huong, Institute of Geodesy and Cartography, Vietnam

Recently, the accuracy of precise point positioning (PPP) has improved significantly thanks to PPP products from several services. These services provide different PPP products in terms of their ability at ambiguity resolution (AR) and the number of available satellite systems. We would like to study how additional non -GPS systems and AR treatment affect PPP accuracy. By processing 24h GNSS data at 47 IGS permanent stations with different options for AR treatment and the combination of different satellite systems, our results show that there is no accuracy improvement on the $24 \mathrm{~h}$ solution $(2.1,2.1,5.8 \mathrm{~mm}$ in North, East and Up components). However the combination of GPS + GLONASS + GALILEO with GPS AR gives the best accuracy on the epoch-by-epoch solution (6.6, $6.2,20.2 \mathrm{~mm}$ ). Compared with the cases of GPS only with AR, this option can improve the accuracy up to $26 \%$ on the Up component. $O$

Keywords: PPP; Multi-GNSS; IGS; ambiguity resolution 